\title{
L'alimentazione "mediterranea" nel paziente con rene policistico
}

\author{
Maria Rosaria Rossetti ${ }^{1}$, Giuseppe Dalfino ${ }^{1}$, Deni Aldo Procaccini ${ }^{2}$, Loreto Gesualdo ${ }^{3}$
}

\author{
${ }^{1}$ Ce.R.T.A. - Centri Regionali per le Tecnologie Agroalimentari, Foggia \\ ${ }^{2}$ Nefrologo, Foggia \\ ${ }^{3}$ Cattedra di Nefrologia, Università degli Studi di Bari, Bari
}

\begin{abstract}
MEDITERRANEAN DIET FOR PATIENTS WITH POLYCYSTIC KIDNEY DISEASE
Abstract. The changes that have occurred in people's eating habits and lifestyle from the second half of the 20th century have modified the state of health of the general population. Extensive scientific evidence indicates the need of reconsidering the eating habits, reverting to the typical foods of the Mediterranean diet. The Mediterranean diet, together with physical activity, abstention from smoking and other addictions, and with conviviality can have an effect not only on the progression of chronic degenerative diseases but also, certainly, on quality of life. It is well known that a high-protein diet with high sodium content, combined with alcohol abuse, is an important cause of the worsening of renal diseases. By contrast, following a Mediterranean diet, rich in fruits, vegetables, legumes, cereals, and olive oil with a moderate consumption of animal proteins and alcohol, plays a protective role on renal function. The Medi $\neg$ terranean diet has proved to contribute to the reduction of cardiovascular diseases and oxidative stress. Hence, there are the conditions to assess whether the Mediterranean diet is able to significantly reduce the activation of the oxidative stress and, therefore, the cardio-vascular risk in patients with ADPKD.
\end{abstract}

Key words: Diet and health, Polycystic kidney disease, ADPKD, Mediterranean Diet, Nutritional epidemiology

Conflict of interest: None.

Ricevuto: 2 Dicembre, 2012; Accettato: 1 Gennaio 2013

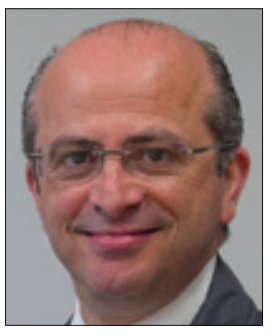

Loreto Gesualdo

Da quando Ancel Keys, con il "Seven Countries Study" (1), confrontò le abitudini alimentari di Italia, Grecia e Jugoslavia con quelle di Stati Uniti, Giappone, Finlandia e Olanda, accendendo i riflettori sul valore della Dieta Mediterranea $(\mathrm{DM})$, già, peraltro, preconizzato dal nutrizionista genovese Luigi Piroddi, vi è stato un crescendo di attenzione verso questo tipo di alimentazione. Più correttamente, a dire il vero, si deve parlare di "stile di vita mediterraneo", in quanto, oltre all'alimentazione, bisogna tenere conto dell'attività fisica, delle attitudini socio-relazionali, delle tradizioni culturali e dell'astensione dal fumo o da altre dipendenze. Il clou è stato raggiunto nel Novembre del 2010 quando l'UNESCO ha inserito la DM nella "Lista del Patrimonio Culturale Immateriale dell'Umanità" e a Pioppi, nel Cilento, dove Keys ha vissuto per molti anni, è stato inaugurato un museo dedicato alla DM. Questo modello alimentare è caratterizzato da un elevato apporto di verdura, da frutta fresca in abbondanza (non disdegnando un consumo controllato di frutta secca, per esempio, mandorle, noci, ecc.), da legumi e cereali, da uno scarso uso di carne rossa, da una discreta quantità di pesce, soprattutto azzurro, e da una moderata assunzione di vino (preferibilmente rosso) durante i pasti e di olio d'oliva come condimento.

Nel corso degli anni sono stati effettuati numerosi studi per valutare l'associazione della DM con diverse patologie (2).

Come affermato da Frank $\mathrm{Hu}$ (3), in antitesi con il tradizionale approccio nutrizionista, che vedrebbe sottostimato il valore della complessa sinergia dei diversi principi nutritivi che, interagendo tra di loro, influenzano fortemente funzioni fisiologiche, metabolismo e assorbimento, viene ormai applicato il concetto di valutazione globale di una dieta (pattern di dieta) e non di singole sostanze. Utilizzando questo razionale, sono stati messi a punto degli indici (score) per valutare in maniera sintetica l'aderenza alla DM classica e i suoi effetti sulla salute: dal valore minimo 0 , che indica una minore corrispondenza, gradualmente si giunge al valore massimo 9, che denota un'alimentazione autenticamente mediterranea. La dieta DASH (Dietary Approaches to Stop Hypertension), oggetto di una ricerca pubblicata nel 2001, ha valutato l'effetto dell'aggiunta di frutta e verdura a una dieta western style accoppiata a differenti quantità di apporto dietetico di $\mathrm{Na}$ in persone con o senza ipertensione arteriosa. I partecipanti selezionati con tecnica random hanno seguito o una tipica dieta di controllo 
(western style) o la DASH (western style + frutta e verdura), suddivisi in tre sottogruppi all'interno della dieta assegnata, in base alla quantità di contenuto di $\mathrm{Na}$ (elevato, intermedio o basso) assunto per 30 giorni consecutivi. Questo lavoro dimostra che le quantità di sodio sono in grado di influenzare in maniera significativa i valori pressori sia nella dieta western style sia nella DASH. Un dato interessantissimo che mette in evidenza questo studio e che merita un approfondimento futuro è, comunque, rappresentato dall'effetto antipertensivo aggiuntivo ottenuto nella dieta DASH, ricca di frutta e verdura. Infatti, è degno di nota osservare che i pazienti sottoposti a dieta DASH presentavano elevati valori di potassiuria dovuti all'assunzione di frutta e verdura, alimenti ricchi di potassio. Questo dato suggerisce che il potassio, più del sodio, gioca un ruolo fondamentale nel controllo pressorio (4).

Sofi et al., nel 2008, hanno effettuato uno studio di metanalisi, selezionando, tra 62 lavori iniziali, 12 studi con una popolazione totale di 1.574.299 soggetti sani, seguiti per un periodo compreso tra i 3 e i 18 anni, rilevando circa 40.000 eventi fatali e non. L'adesione alla DM, valutata tramite score, si associava significativamente a una riduzione del rischio di mortalità in generale $(9.1 \%)$ e per malattia cardiovascolare $(9.4 \%)$, all'incidenza del cancro e alla mortalità $(9 \%)$ e all'incidenza della malattia di Parkinson e della malattia di Alzheimer (8.7\%) (5). Lo stesso gruppo di studio nel 2010 ha aggiornato il lavoro sopracitato, ampliando la popolazione dello studio. Sono stati confermati i risultati precedenti ed è stato rilevato l'effetto protettivo della DM sull'incidenza e sulla mortalità nelle malattie cronico-degenerative, con estensione all'ictus e alle forme di deterioramento cognitivo lieve (6).

L'acido alfa-linolenico (ALA), abbondante in particolare in mandorle e noci, concorre, verosimilmente, alle proprietà cardioprotettive della DM (7). ALA è un acido grasso omega-3 (18:03 n-3) di tipo "essenziale", sintetizzato dall'uomo, ma introdotto tramite gli alimenti. Le fonti alimentari primarie di ALA sono gli alimenti vegetali e, pertanto, una dieta ricca di verdure verdi, frutta e semi (mandorle, noci, ecc.), come la DM, rappresenta un'ottima fonte di questo fattore e di grandi quantità di potassio. Diversi studi, sia studi prospettici che trial clinici randomizzati (8-13), suggeriscono che l'ALA di provenienza alimentare abbia un effetto benefico sul sistema cardiovascolare.

ALA è il precursore di tutti gli altri n-3 acidi grassi polinsaturi a catena lunga, tra cui l'acido eicosapentaenoico (EPA), l'acido docosapentaenoico (DPA) e l'acido docosaesaenoico (DHA). ALA, di per sé, e i suoi derivati, tra cui le prostaglandine della serie 3 e i leucotrieni della serie 5, hanno proprietà anti-infiammatorie, anti-trombotiche e anti-aritmiche. Un'elevata assunzione di ALA inibisce anche la produzione di leucotrieni della serie 4 e la produzione di prostaglandine, leucotrieni e trombossani della serie 2 , tutti potenti mediatori proinfiammatori e pro-trombotici. Pertanto, l'assunzione alimentare di ALA può ridurre l'infiammazione sistemica, ridurre la trombofilia e stabilizzare le proprietà elettriche del cuore (7). Lo studio ATTICA (14), in un periodo di osservazione di cinque anni, ha valutato l'eventuale associazione tra adesione alla DM e livelli di funzione renale in 1975 soggetti non affetti da patologia cardiovascolare e/o da ipertensione arteriosa, se- lezionati in maniera randomizzata secondo la distribuzione di età e sesso della popolazione residente nella regione ATTICA della Grecia. L'adesione alla DM è stata valutata tramite uno specifico questionario validato (MedDietScore), il cui punteggio varia teoricamente da 0 a 55 (a valori più alti corrisponde una maggiore aderenza alla DM) (15), e la funzione renale è stata espressa in termini di Clearance della creatinina misurata con la formula di Cockcroft-Gault (16). Il disegno dello studio ha previsto una valutazione basale e una valutazione successiva dopo 5 anni di follow-up.

Alla valutazione basale, l'associazione tra l'adesione alla $\mathrm{DM}$ e la funzione renale risultava positivamente significativa $(\mathrm{p}<0.001)$ all'analisi univariata. Il trend positivo di tale associazione veniva confermato anche all'analisi multivariata, senza raggiungere la significatività statistica $(\mathrm{p}<0.06)$.

È noto che la dieta iperproteica a elevato contenuto di sodio, unitamente all'abuso di alcol, costituisce un determinante della progressione della malattia renale (17). Al contrario, l'adesione alla DM, ricca di frutta, verdura, legumi, cereali e olio d'oliva e moderata nel consumo di proteine animali e alcool, gioca un ruolo protettivo della funzione renale (Tab. I).

Innumerevoli studi condotti su modelli animali hanno indotto a ritenere lo stress ossidativo un fenomeno importante nella patogenesi di molte malattie legate all'invecchiamento, comprese le malattie cardiovascolari (18). Le reazioni ossidative fanno parte del normale processo di produzione di energia durante il metabolismo aerobico e generano specie reattive dell'ossigeno, che possono essere deleterie, se non controllate. Tuttavia, queste molecole svolgono anche azioni biologiche utili, tra cui funzioni di biosintesi e di disintossicazione. La vita aerobica ha sviluppato una serie complessa di sistemi antiossidanti per controllare queste reazioni e riparare o sostituire apparati danneggiati.

La definizione di stress ossidativo è stata di recente riformulata, considerandolo non solo un semplice squilibrio globale di pro-ossidanti e antiossidanti, ma anche una condizione di perturbazione di segnali redox e di specifiche vie di controllo (19). Ciò aiuta a comprendere perché per lungo tempo gli studi interventistici su larga scala in esseri umani non sono stati in grado di cogliere i benefici dell'uso di supplementi antiossidanti sulla salute e sugli esiti della malattia. I meccanismi molecolari alla base delle vie di trasduzione del segnale redox che determinano la differente risposta cellulare avvengono attraverso percorsi redox all'interno delle stesse cellule, per cui lo stress ossidativo potrebbe avvenire senza uno squilibrio generale di pro-ossidanti e antiossidanti. Inoltre, dato che i sistemi di segnale e di controllo redox sono meccanismi omeostatici fondamentali in molti processi cellulari, $i$ danni agli apparati

TABELLA I - ADESIONE ALLA DIETA MEDITERRANEA E FUNZIONE RENALE

\begin{tabular}{lcccc}
\hline & $\begin{array}{c}\text { Clear. Creat. } \\
>\mathbf{9 0} \mathbf{~ m L / m},\end{array}$ & $\begin{array}{c}\text { Clear. Creat. } \\
\mathbf{6 0 - 9 0} \mathbf{~ m L / m},\end{array}$ & $\begin{array}{c}\text { Clear. Creat. } \\
<\mathbf{6 0} \mathbf{~ m L / m},\end{array}$ & $\mathbf{p}$ \\
\hline $\begin{array}{l}\text { MedDietScore } \\
(0-55)\end{array}$ & $27(6)$ & $28(7)$ & $24(5)$ & $<0.001$ \\
\hline
\end{tabular}


macromolecolari non sono l'unico modo con cui lo stress ossidativo può causare malattie. Altri meccanismi comprendono, per esempio, l'embriogenesi, l'apoptosi, un alterato controllo del ciclo cellulare, disfunzioni immunitarie, processi fibrotici e un'alterata permeabilità di membrana. Nei pazienti affetti da malattia renale policistica (ADPKD), gli eventi maggiori sono rappresentati dalla progressione dell'insufficienza renale e dall'aumentato rischio cardiovascolare; la causa di morte più frequente $(80 \%)$ è attribuibile alla malattia coronarica $(20$, 21). Nella patogenesi di tali complicanze gioca un ruolo importante l'attivazione dello stress ossidativo. I dati relativi allo stress ossidativo in pazienti con ADPKD sono limitati. Maser et al. (22) hanno studiato l'espressione genica di marker dello stress ossidativo in un modello di ratto con ADPKD. Essi hanno rilevato un aumento della trascrizione dell'eme-ossigenasi-1 e una riduzione dell'espressione genica degli enzimi antiossidanti, che si correlavano con la severità della malattia. Inoltre, hanno rilevato anche una riduzione quali-quantitativa degli enzimi antiossidanti glutatione perossidasi e superossidodismutasi con conseguente accumulo di prodotti di degradazione della perossidazione lipidica nel plasma e nei reni. Uno studio di Wang et al. ha rilevato in 27 pazienti, affetti da ADPKD in fase iniziale, elevati livelli plasmatici e urinari di acido 13-idrossioctadecadienoico, marker dello stress ossidativo (23). Infine, la presenza di stress ossidativo è stata rilevata dagli elevati livelli plasmatici di 8-epi-PGF2 e dalla riduzione di SDO in 144 pazienti con ADPKD, ipertesi e non, e con vari livelli di funzione renale (24).

È noto che i polifenoli naturalmente presenti nei cibi mediterranei hanno chiare proprietà antiossidanti $(25,26)$. Anche se le proprietà antiossidanti della DM sono note, le indagini dirette sulla modulazione dello stress ossidativo nell'uomo sono poche e limitate. Pochi studi (27-29) hanno esaminato gli effetti della DM sui vari biomarcatori sierici di stress ossidativo; in quello più rappresentativo (30), i soggetti assegnati alla DM presentavano livelli più bassi di LDL ossidate, se paragonati ai soggetti della dieta di controllo. Nel campione di coppie di gemelli, che differivano per l'adesione alla $\mathrm{DM}$, è stata trovata una robusta associazione inversa tra il punteggio DM e stress ossidativo (31). Lo stress ossidativo è stato misurato con il rapporto GSH/GSSG e il risultato si è dimostrato indipendente da una vasta gamma di fattori noti di rischio cardiovascolare. La differenza di un'unità nel punteggio della DM era associata a un aumento del 10\% del rapporto GSH/GSSG (che denota un ridotto stress ossidativo) all'interno delle coppie di gemelli, confrontando il gemello con un punteggio maggiore per adesione alla DM con il suo gemello con un punteggio inferiore. Benché siano necessari studi clinici più vasti e disegnati meglio, con modificazioni della dieta a lungo termine, questo studio suggerisce che una dieta abituale, con un profilo che si avvicina alla DM, è associata a un favorevole equilibrio metabolico-ossidativo. Da quanto rilevato in letteratura, la DM è in grado di ridurre malattie cardiovascolari e stress ossidativo. Esistono i presupposti, quindi, per una ricerca nefrologica che deve mirare a valutare se la DM sia in grado di ridurre in maniera significativa l'attivazione dello stress ossidativo in pazienti con ADPKD e, quindi, il loro rischio cardiovascolare.

\section{Riassunto}

Dalla seconda metà del XX secolo sono intervenute modificazioni nelle abitudini alimentari e nello stile di vita, tali da determinare variazioni anche nello stato di salute della popolazione. Numerose evidenze scientifiche indicano la necessità di dover rivedere l'alimentazione, ritornando a preferire i cibi tipici della Dieta Mediterranea, che, unitamente all'attività fisica, all'astensione dal fumo o da altre dipendenze e alla convivialità, possono influire anche sulla progressione delle malattie cronico-degenerative e certamente sulla Qualità della Vita. È noto che la dieta iperproteica a elevato contenuto di sodio, unitamente all'abuso di alcool, costituisce un determinante della progressione della malattia renale. Al contrario, l'adesione alla Dieta Mediterranea, ricca di frutta, verdura, legumi, cereali e olio d'oliva e moderata nel consumo di proteine animali e alcool, gioca un ruolo protettivo della funzione renale. La Dieta Mediterranea si è mostrata in grado di ridurre malattie cardiovascolari e stress ossidativo, pertanto esistono i presupposti per valutare se l'alimentazione di tipo mediterraneo sia in grado di ridurre in maniera significativa l'attivazione dello stress ossidativo anche in pazienti con ADPKD e, quindi, il loro rischio cardiovascolare.

Parole chiave: Dieta e salute, Malattia policistica renale, ADPKD, Dieta Mediterranea, Epidemiologia nutrizionale

Dichiarazione di conflitto di interessi: Gli Autori dichiarano di non avere conflitto di interessi.

Indirizzo degli Autori:

Prof. Loreto Gesualdo

Università degli Studi di Bari

Direzione S.C. Nefrologia, Dialisi e Trapianto

Azienda Ospedaliero-Universitaria Consorziale "Policlinico" Piazza Giulio Cesare 11

70124 Bari

loreto.gesualdo@uniba.it 


\section{Bibliografia}

1. Keys AE, Blackburn HMA, Buzina R, et al. Coronary heart disease in seven countries. Circulation 1970; 41 (Suppl. 1): 1-211.

2. Kris-Etherton P, Eckel RH, How-ard BV, et al. Diet Heart Study. Benefits of a Mediterranean-style, National Cholesterol Education Program/American Heart Association Step I Dietary Pattern on Cardiovascular Disease. Circulation 2001; 103 (13): 1823-5.

3. Hu FB. Dietary pattern analysis: a new direction in nutritional epidemiology. Curr Opin Lipidol 2002; 13 (1): 3-9.

4. Sacks FM, Svetkey LP, Vollmer WM, et al. Effects on blood pressure of reduced dietary sodium and the Dietary Approaches to Stop Hypertension (DASH) diet. DASH-Sodium Collaborative Research Group. N Engl J Med 2001; 344: 3-10.

5. Sofi F, Cesari F, Abbate R, et al. Adherence to Mediterranean diet and health status: meta-analysis. BMJ 2008; 337: a1344.

6. Sofi F, Abbate R, Gensini GF, Casini A. Accruing evidence on benefits of adherence to the Mediterranean diet on health: an updated systematic review and meta-analysis. Am J Clin Nutr 2010; 92: 1189-96.

7. Mozaffarian D. Does alpha-linolenic acid intake reduce the risk of coronary heart disease? A review of the evidence. Altern Ther Health Med 2005; 1: 24-30.

8. Djousse L, Folsom AR, Province MA, Hunt SC, Ellison RC. Dietary linolenic acid and carotid atherosclerosis: the National Heart, Lung, and Blood Institute Family Heart Study. Am J Clin Nutr 2003; 77: 819-825.

9. Albert CM, Oh K, Whang W, et al. Dietary alpha-linolenic acid intake and risk of sudden cardiac death and coronary heart disease. Circulation 2005; 112: 3232-8.

10. Singh RB, Dubnov G, Niaz MA, et al. Effect of an Indo-Mediterranean diet on progression of coronary artery disease in high risk patients (Indo-Mediterranean Diet Heart Study): a randomised single-blind trial. Lancet 2002; 360 (9344): 1455-61.

11. de Lorgeril M, Salen P, Martin JL, et al. Mediterranean diet, traditional risk factors, and the rate of cardiovascular complications after myocardial infarction: final report of the Lyon Diet Heart Study. Circulation 1999; 99: 779-85.

12. Ascherio A, Rimm EB, Giovannucci EL, Spiegelman D, Stampfer M, Willett WC. Dietary fat and risk of coronary heart disease in men: cohort follow up study in the United States. BMJ 1996; 313: 84-90.

13. Mozaffarian D, Ascherio A, Hu FB, et al. Interplay between different polyunsaturated fatty acids and risk of coronary heart disease in men. Circulation 2005; 111: 157-64.

14. Chrysohoou C, Panagiotakos DB, Pitsavos C, et al. Renal function, cardiovascular disease risk factors' prevalence and 5-year disease incidence; the role of diet, exercise, lipids and inflammation markers: the ATTICA study. QJM 2010; 103: 413-22.

15. Panagiotakos DB, Pitsavos C, Stefanadis C. Dietary patterns:a Mediterranean diet score and its relation to clinical and biological markers of cardiovascular disease risk. Nutr Metab Cardiovasc Dis 2006; 6: 559-68.
16. Cockcroft DW, Gault MH. Prediction of creatinine clearance from serum creatinine. Nephron 1976; 16: 31-41.

17. O'Meara E, Chong KS, Gardner RS, Jardine AG, Neilly $\mathrm{JB}, \mathrm{McD}$ onagh TA. The modification of diet in renal disease (MDRD) equations provide valid estimations of glomerular filtration rates in patients with advanced heart failure. Eur J Heart Fail 2006; 8: 63-7.

18. Madamanchi NR, Vendrov A, Runge MS. Oxidative stress and vascular disease. Arterioscler Thromb Vasc Biol 2005; 25 (1): 29-38.

19. Jones DP. Redefining oxidative stress. Antioxid Redox Signal 2006; 8: 1865-79.

20. Perrone RD, Ruthazer R, Terrin NC. Survival after endstage renal disease in autosomal dominant polycystic kidney disease: Contribution of extrarenal complications to mortality. Am J Kidney Dis 2001; 38: 777-84.

21. Fick GM, Johnson AM, Hammond WS, Gabow PA. Causes of death in autosomal dominant polycystic kidney disease. J Am Soc Nephrol 1995; 5: 2048-56.

22. Maser RL, Vassmer D, Magenheimer BS, et al. Oxidant stress and reduced antioxidant enzyme protection in polycystic kidney disease. J Am Soc Nephrol 2002; 13: 991-9.

23. Wang D, Strandgaard S, Borresen ML, et al. Asymmetric dimethylarginine and lipid peroxidation products in early autosomal dominant polycystic kidney disease. Am J Kidney Dis 2008; 51: 184-91.

24. Menon V, Rudym D, Chandra P, Miskulin D, Perrone R, Sarnak $M$. Inflammation, oxidative stress, and insulin resistance in polycystic kidney disease. Clin J Am Soc Nephrol 2011; 6: 7-13.

25. Alvarez P, Alvarado C, Mathieu F, Jimenez L, De la Fuente M. Diet supplementation for 5 weeks with polyphenol-rich cereals improves several functions and the redox state of mouse leucocytes. Eur J Nutr 2006; 45: 428-38.

26. Davis L, Stonehouse W, Loots du T, et al. The effects of high walnut and cashew nut diets on the antioxidant status of subjects with metabolic syndrome. Eur J Nutr 2007; 46: 155-64.

27. Ambring A, Friberg P, Axelsen M, et al. Effects of a Mediterranean-inspired diet on blood lipids, vascular function and oxidative stress in healthy subjects. Clin Sci (Lond) 2004; 106 : 519-25.

28. Stachowska E, Wesolowska T, Olszewska M, et al. Elements of Mediterranean diet improve oxidative status in blood of kidney graft recipients. Br J Nutr 2005; 93: 345-352.

29. Hagfors L, Leanderson P, Skoldstam L, Andersson J, Johansson G. Antioxidant intake, plasma antioxidants and oxidative stress in a randomized, controlled, parallel, Mediterranean dietary intervention study on patients with rheumatoid arthritis. Nutr $\mathrm{J}$ 2003; $2: 5$.

30. Fito M, Guxens M, Corella D, et al. Effect of a traditional Mediterranean diet on lipoprotein oxidation: a randomized controlled trial. Arch Intern Med 2007; 167: 1195-203.

31. Dai J, Jones DP, Goldberg J, et al. Association between adherence to the Mediterranean diet and oxidative stress. Am J Clin Nutr 2008; 88: 1364-70. 